\title{
Characterization of the nucellus-specific dehydrin MdoDHN11 demonstrates its involvement in the tolerance to water deficit
}

 \\ Araújo Mariath $^{3} \cdot$ Giancarlo Pasquali $^{1} \cdot$ Márcia Margis-Pinheiro $^{1} \cdot$ Luís Fernando Revers $^{1,2}$
}

Received: 7 March 2019 / Accepted: 18 May 2019

○) Springer-Verlag GmbH Germany, part of Springer Nature 2019

\begin{abstract}
Key message MdoDHN11 acts in the nucellus layer to protect the embryo and the endosperm from limited water availability during apple seed development.

Abstract Dehydrins (DHNs) are protective proteins related to several plant developmental responses that involve dehydration such as seed desiccation and abiotic stresses. In apple (Malus $\times$ domestica Borkh.), the seed-specific MdoDHN11 was suggested to play important roles against dehydration during seed development. However, this hypothesis has not yet been evaluated. Within this context, several experiments were performed to functionally characterize MdoDHN11. In situ hybridization analysis during apple seed development showed that MdoDHN11 expression is confined to a maternal tissue called nucellus, a central mass of parenchyma between the endosperm and the testa. The MdoDHN11 protein was localized in the cytosol and nucleus. Finally, transgenic Arabidopsis plants expressing MdoDHN11 were generated and exposed to a severe water-deficit stress, aiming to mimic a situation that can occurs during seed development. All transgenic lines showed increased tolerance to water deficit in relation to wild-type plants. Taken together, our results provide evidences that MdoDHN11 plays important roles during apple seed development by protecting the embryo and the endosperm from limited water availability, and the mechanism of action probably involves the interaction of MdoDHN11 with proteins and other components in the cell.
\end{abstract}

Keywords Apple $\cdot$ Dehydrin $\cdot$ Seed development $\cdot$ Transgenic expression $\cdot$ Water-deficit stress

Communicated by Zheng-Yi Xu.

Electronic supplementary material The online version of this article (https://doi.org/10.1007/s00299-019-02428-8) contains supplementary material, which is available to authorized users.

Luís Fernando Revers

luis.revers@embrapa.br

1 Graduate Program in Cell and Molecular Biology, Centro de Biotecnologia, Universidade Federal do Rio Grande do Sul (UFRGS), Porto Alegre 91501-970, Brazil

2 Embrapa Uva e Vinho, Rua Livramento, 515, P.O. Box 130, Bento Gonçalves, RS 95701-008, Brazil

3 Laboratory of Plant Anatomy, Botany Department, UFRGS, Porto Alegre 91501-970, Brazil

4 Present Address: AGAP, Univ. Montpellier, CIRAD, INRA, Montpellier SupAgro, Montpellier, France

\section{Introduction}

Seeds are long-term storage structures commonly used by plants to propagate their descendants, given that these structures maintain their viability even under adverse environmental conditions. In flowering plants, the achievement of normal seed development is dependent on the coordination of growth and differentiation between three genetically different components: maternal tissues, embryo and endosperm (Hartmann et al. 2010). The last two are originated by the double fertilization of the egg and the central cell in the female gametophyte, and are responsible for the initiation of seed development (Sreenivasulu and Wobus 2013; Bleckmann et al. 2014). Although the maternal tissues of the ovule do not participate in the fertilization process, they undergo several reprogramming events in response to it (Sreenivasulu and Wobus 2013). One of these maternal tissues is a central mass of parenchyma cells called nucellus, which has nutritive and protective roles (Hartmann et al. 2010). During 
angiosperm seed evolution, plants have shifted between endosperm and nucellus as the main nutrient storage system (Xu et al. 2016). To reach physiological maturity, seeds need to acquire tolerance to desiccation, a process that involves critical water losses to prepare the seed for germination (Hartmann et al. 2010). For the proper seed development, several molecules are recruited to protect different seed components (González-Morales et al. 2016).

One of the protective proteins that stand out in the process of seed development includes a class of polypeptides belonging to the group II of late embryogenesis abundant (LEA) proteins called dehydrins (DHNs) (Banerjee and Roychoudhury 2016). These proteins are characterized by the presence of a highly conserved domain, the K-segment, followed or not by two other well-conserved domains, the $\mathrm{Y}$ - and S-segments. Under stress conditions, the $\mathrm{K}$-segment is able to form an $\alpha$-helical structure, which helps the stabilization of proteins and cellular membranes (Liu et al. 2017). Indeed, DHNs play important roles in plant developmental responses that involve dehydration tolerance such as seed desiccation and abiotic stresses (Hanin et al. 2011). Although many putative functions were reported for DHNs such as radical-scavenging activity and binding to membranes, metals, lipids and DNA, their precise mechanisms of action remain unknown (reviewed in Hanin et al. 2011; Graether and Boddington 2014; Liu et al. 2017). Given that DHNs are usually classified as intrinsically disordered proteins (IDPs), their function is commonly associated with this feature (Tompa et al. 2006). Their structure is prone to act as water replenishers and prevent an adverse increase in ionic strength, allowing DHNs to interact and protect membranes and other proteins during dehydration (Tompa et al. 2006; Banerjee and Roychoudhury 2016; Riley et al. 2019).

In apple (Malus $\times$ domestica Borkh.), one of the most economically important perennials worldwide, a high functional diversification was identified among its $11 \mathrm{DHN}$ $(M d o D H N)$ genes. Based on the presence of conserved domains ( $\mathrm{K}, \mathrm{Y}$ and $\mathrm{S}$ ), MdoDHNs were assigned into four out of five DHN subclasses (Falavigna et al. 2015). $M d o D H N$ genes were characterized according to their transcript expression in response to drought, cold, and abscisic acid treatment (Liang et al. 2012), as well as during bud dormancy progression, flowering and fruit ripening stages (Falavigna et al. 2015). However, no apple $D H N$ was functionally characterized so far. MdoDHN11, a $\mathrm{Y}_{3} \mathrm{SK}_{2}$ dehydrin, is an interesting candidate to be further characterized due to its seed-specific transcript accumulation pattern and its probable protective role during apple seed tolerance to desiccation (Falavigna et al. 2015).

In the present work, the functional characterization of the MdoDHN11 gene was carried out. To accomplish this goal, the spatio-temporal MdoDHN11 gene expression was analyzed in apple seeds, the subcellular localization of the encoded protein was demonstrated, as well as the performance of transgenic Arabidopsis plants expressing MdoDHN11 under a severe water-deficit stress. These assays were performed aiming to better understand the functional role of $M d o D H N 11$, and the data gathered in this work confirmed its protective relevance during long-term water deficit, in a similar manner to a situation that can occurs during seed development.

\section{Materials and methods}

\section{Plant material}

Apple fruits were harvested in an experimental orchard located at Embrapa Uva e Vinho (- 29.165762, - 51.535147 and $614 \mathrm{~m}$ above sea level) in Southern Brazil. Apple trees were maintained using standard orchard management practices. Plant material consisted of 2-year-old 'Imperial Gala' trees grafted on M.7 rootstocks. Flowers at the anthesis stage were tagged in the field. Apple fruits were harvested at 22, 51, and 83 days after anthesis (DAA), which corresponded to fruits with 20, 40, and $60 \mathrm{~mm}$ diameter, respectively (Fig. 1a, $\mathrm{d}, \mathrm{g})$. At each sampling point, at least six individual whole fruits were analyzed. Seeds were immediately removed and fixed in $4 \%$ formaldehyde under vacuum $(600 \mathrm{mmHg})$ for 30 min followed by overnight incubation at $4{ }^{\circ} \mathrm{C}$ (McDowell and Trump 1976). Samples were dehydrated through a graded series of ethyl alcohol from 30 to $100 \%$ and stored at $-20{ }^{\circ} \mathrm{C}$ until use.

\section{Seed morphological assay}

The fixed and dehydrated seed samples were embedded in 2-hydroxyethyl methacrylate resin as described in Gerrits and Smid (1983). The $5 \mu \mathrm{m}$ sections were obtained using a Leica RM 2255 microtome. The metachromatic reagent Toluidine Blue $\mathrm{O}$ was used to stain seed structures by submerging the slides with sections in the reagent for $1 \mathrm{~min}$ (Feder and O'brien 1968). The stained tissues were photomicrographed in bright field using a Leica DM1000 LED microscope coupled with a Leica MC170 HD camera system. Photomicrographs were merged and the background color was adjusted to white.

\section{Nucleic acid extraction and cDNA synthesis}

DNA was purified from young leaves $(100 \mathrm{mg})$ using modified protocols scaled down to $2 \mathrm{~mL}$ centrifuge tubes (Lodhi et al. 1994; Lefort and Douglas 1999). Total RNA was isolated (Zeng and Yang 2002; Falavigna et al. 2014) and DNase-treated using the TURBO DNA-free Kit (Ambion). The SuperScript ${ }^{\mathrm{TM}}$ III Reverse Transcriptase (Thermo Fisher 
Fig. 1 Morphoanatomical analysis of seeds during apple development. a-c $20 \mathrm{~mm}$ fruits (22 DAA). d-f $40 \mathrm{~mm}$ fruits (51 DAA). $\mathbf{g}-\mathbf{i} 60 \mathrm{~mm}$ fruits ( 83 DAA). Representative images of the used apples are present in a, $\mathbf{d}$ and $\mathbf{g}$, with a scale bar equal to $1 \mathrm{~cm}$. Light micrographs of apple seed cross sections stained with Toluidine blue $\mathrm{O}$ are shown in $\mathbf{b}, \mathbf{c}, \mathbf{e}, \mathbf{f}, \mathbf{h}$ and i, with a scale bar of $500 \mu \mathrm{m}$. Enlarged images of the peripheral seed structure are presented in c, $\mathbf{f}$ and i. $E N$ endosperm, $E M$ embryo, $N U$ nucellus, $T E$ testa (color figure online) a
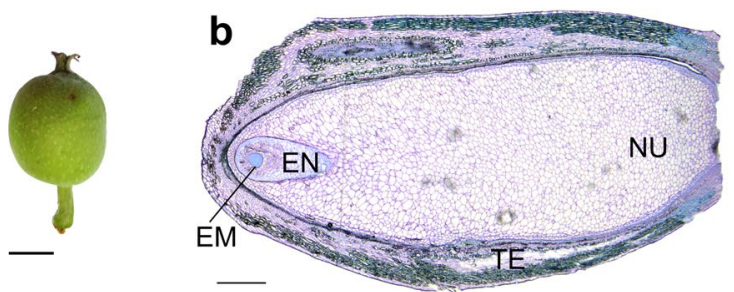

d

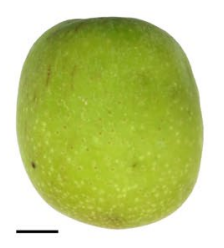

e

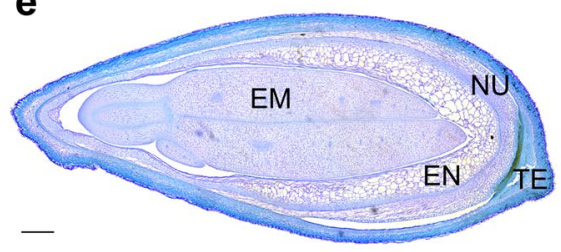

g
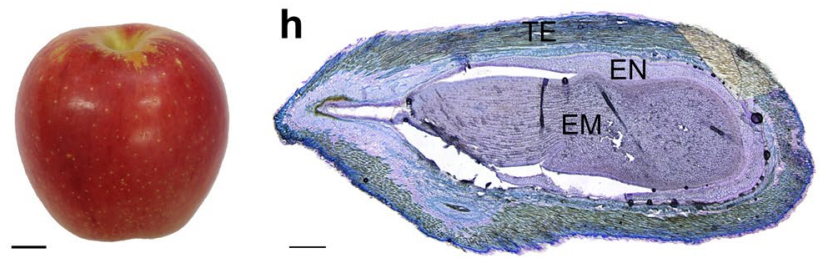
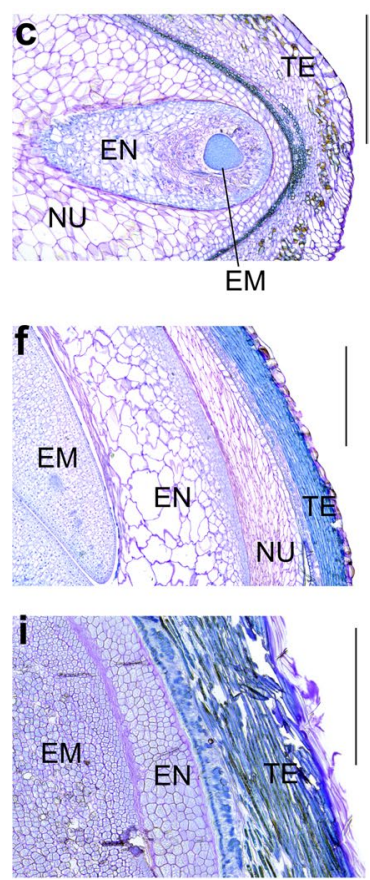

Scientific) was used for cDNA synthesis, according to manufacturer's instructions.

\section{In situ hybridization}

Seed samples were embedded in paraffin and longitudinal and transversal sections $(8-10 \mu \mathrm{m})$ were prepared and mounted on silanized microscope slides as described (Malabarba et al. 2017). A gene-specific fragment of $225 \mathrm{bp}$ (MdoDHN11_ISH_probe in Supplementary Table 1) from MdoDHNI1 was amplified from cDNA obtained from seeds of 'Imperial Gala' fruits and cloned into the $\mathrm{pGEM}^{\circledR}$-T Easy vector (Promega), according to manufacturer's instructions. The resulting recombinant vector was confirmed by sequencing. Sense and antisense labeled probes were generated by digoxigenin-labeling using the DIG RNA Labeling Kit (SP6/ T7) (Roche). After detection of the hybridization signals by immunostaining, slides were washed, dehydrated, and mounted using Entellan ${ }^{\circledR}$ (Merck). Photomicrographs were obtained as described above.

\section{Subcellular localization of MdoDHN11}

To identify the subcellular localization of MdoDHN11, a transient expression assay in Arabidopsis thaliana ecotype Columbia-0 (Col-0) leaf protoplasts was performed. The complete coding sequence of MdoDHN11 was amplified using Platinum ${ }^{\circledR} P f x$ DNA polymerase (Thermo Fisher Scientific) from cDNA obtained from 'Imperial Gala' seeds using gene-specific primers (Supplementary Table 1). The amplicon was cloned into pENTR ${ }^{\mathrm{TM}}$ Directional
$\mathrm{TOPO}^{\circledR}$ (Thermo Fisher Scientific), generating the TOPO$M d o D H N 11$ construct. This construct was used to fuse the coding sequence of EYFP (enhanced yellow fluorescent protein) to the 5' portion of MdoDHN11 using the p2YGW7 vector (Karimi et al. 2007) and Gateway ${ }^{\circledR}$ LR Clonase ${ }^{\mathrm{TM}}$ II Enzyme Mix (Thermo Fisher Scientific). The resulting vector with the 35S::EYFP-MdoDHN11 insert was used in protoplast transformation. Protoplast isolation and transformation were performed essentially as described in $\mathrm{Wu}$ et al. (2009). Negative controls were composed of untransformed cells, whereas positive controls were cells transformed with 35S::EGFP (enhanced green fluorescent protein). Transformed protoplasts and negative controls were incubated under light for $20 \mathrm{~h}$ at $24{ }^{\circ} \mathrm{C}$ prior to imaging. Cell nuclei were stained using $1 \mu \mathrm{g} / \mathrm{mL}$ DAPI (4',6-diamidino2-phenylindole). Fluorescence microscopy was performed using an Olympus FluoView 1000 confocal laser-scanning microscope (UFRGS Electron Microscopy Center, Porto Alegre, Brazil) equipped with a set of filters capable of distinguishing chlorophyll autofluorescence, DAPI, GFP and YFP fluorescence. The assay was repeated twice to confirm the obtained results.

\section{Arabidopsis transformation}

To generate transgenic Arabidopsis plants expressing $M d o D H N 11$, the TOPO-MdoDHN11 construct previously obtained was used to clone MdoDHN11's CDS into the pH7WG2D. 1 vector under CaMV 35S promoter (Karimi et al. 2007) using Gateway ${ }^{\circledR}$ technology (Thermo Fisher Scientific). The resulting vector harboring the $35 \mathrm{~S}:: M d o D H N 11$ 
cassette was confirmed by sequencing and used in the transformation of Agrobacterium tumefaciens (strain EHA105). Wild-type Arabidopsis Col-0 plants were transformed using the floral dip method (Clough and Bent 1998). Transformed seeds were sown in individual plastic pots. Given that the vector encodes EGFP under the control of the rolD promoter (Karimi et al. 2007), a GFP fluorescence screening was performed in 3-week-old plants using a Leica M165FC stereomicroscope. Plants lacking GFP fluorescence were discarded. Total RNA was isolated from leaves and cDNA was synthesized as previously described. To assess transgene expression, real-time PCR was performed using MdoDHN11 gene-specific primers (Supplementary Table 1) as described Falavigna et al. (2014, 2015). Actin 2 (AtAct2) and constitutive photomorphogenic 1 ( $A t C O P 1)$ were used as reference genes (Supplementary Table 1). Amplicons from each line were sequenced to confirm their transgenic nature. Plants without transgene expression were also discarded. Nine independent lines were obtained and further analyzed.

\section{Water-deficit stress}

A preliminary water withhold assay was performed to determine how long wild-type Arabidopsis plants could survive without water in our growth conditions (Falavigna et al. 2018). Wild-type plants were unable to recover after 25 days without water (Falavigna et al. 2018). In the present work, pot capacity of wild-type and transgenic T1 line plants was achieved and maintained prior to the water deficit treatment. All pots have had their weight adjusted to approximately $160 \mathrm{~g}$ (soil, plant, pot and water) before starting the stress assay. The water deficit experiment was performed by transferring unstressed 2-month-old transgenic and wild-type plants to trays without water for 42 days. During the treatment, all pots were independently weighed once a week to measure water loss. After 6 weeks of treatment, water was added to the trays, allowing water influx to the pots through diffusion. Plant recovery was evaluated after 7 days of rehydration. Pictures were taken for visual analysis.

\section{Results}

\section{Morphoanatomical analysis of apple seeds}

Morphoanatomical analyses of 'Imperial Gala' seeds were performed to better characterize the apple seed structure during its development. During the early development of the seed (seeds from $20 \mathrm{~mm}$ fruits), the nucellar tissue increased and the seed coat (testa) differentiated, while the young embryo (globular embryo) remained immersed in the developing endosperm (Fig. 1b, c). In seeds from $40 \mathrm{~mm}$ fruits, the further development of the endosperm and the embryo (torpedo embryo) was observed, limiting the nucellus to a small number of cell layers in contact with the integument already differentiated in the seed coat, with mesotestal characteristics (Fig. 1e, f). In seeds from $60 \mathrm{~mm}$ fruits, a well-developed embryo was observed and the endosperm was reduced to a few cell layers in contact with the testa (Fig. 1h, i).

\section{Spatial and temporal accumulation of MdoDHN11 transcripts and subcellular localization of the encoded protein}

Transcript accumulation of MdoDHNII was previously analyzed in apple during bud dormancy, flowering and fruit development, and its main expression was identified in seeds (Falavigna et al. 2015). Gene expression data from this previous work was reanalyzed aiming to identify which $M d o D H N$ gene presented the highest steady-state mRNA levels during apple seed development (Supplementary Fig. 1). When considering seeds from $40 \mathrm{~mm}$ fruits, MdoDHN11 presented the highest transcript levels among all $M d D H N$ genes. This result prompted us to further characterize the MdoDHN11 spatial and temporal expression patterns using in situ hybridization (ISH). The antisense MdoDHN11 probe signal was clearly visible in the nucellus layer in seeds from $40 \mathrm{~mm}$ fruits (Fig. 2a). ISH slides hybridized with the sense MdoDHN11 probe were used as control and showed no hybridization signals (Fig. 2b).

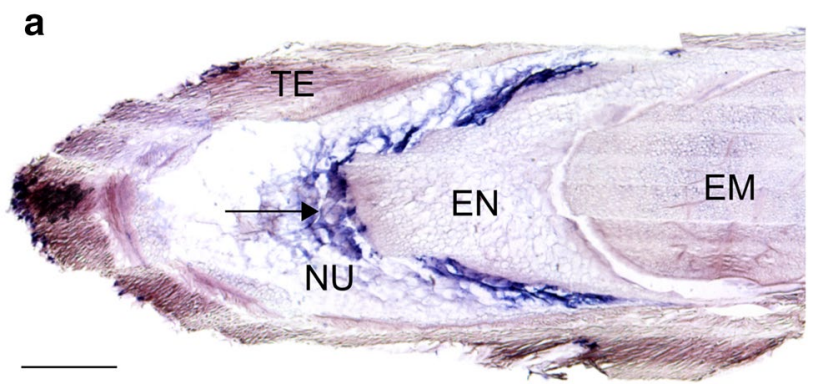

b

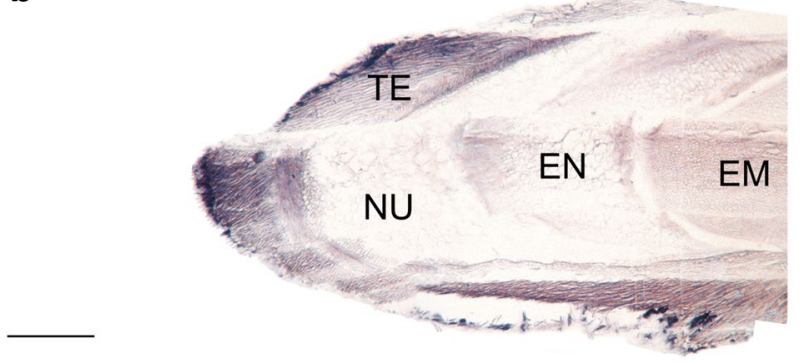

Fig. 2 Spatial and temporal accumulation of MdoDHN11 transcripts in apple seeds. Hybridization signal (black arrow) is visible in the nucellus layer (a) compared to the control slide (b). $E N$ endosperm, $E M$ embryo, $N U$ nucellus, $T E$ testa. Scale bar $500 \mu \mathrm{m}$ (color figure online) 
To gain insights about MdoDHN11 function, EYFP was fused to MdoDHN11 to identify its subcellular localization. Transient expression analyses in Arabidopsis leaf protoplasts revealed that MdoDHN11 was present in the nucleus and cytosol (Fig. 3). Positive controls showed similar subcellular localization, whereas negative controls showed only DAPI and chlorophyll fluorescence.

\section{MdoDHN11-expressing Arabidopsis plants and evaluation of water deficit tolerance}

MdoDHN11 transcript accumulation during apple seed development suggested an adaptive role against dehydration (Falavigna et al. 2015), which is characteristic during the acquisition of seed desiccation tolerance (González-Morales et al. 2016). Aiming to test if the function of MdoDHN11 may be related to protection against dehydration, transgenic Arabidopsis plants expressing MdoDHN11 were generated and submitted to a severe water-deficit stress. Nine independent transgenic lines expressing MdoDHN11 were obtained and analyzed. All transgenic plants expressed the EGFP reporter gene as well as the MdoDHN11 transgene (Fig. 4a). Transformed and wild-type plants were grown under normal conditions for 2 months and then their water supply was removed. Wild-type plants displayed a higher water loss rate during water-deficit stress than transgenic lines, reaching almost half of the initial water soil content in the end of the treatment (Fig. 5). Similar water losses were identified among transgenic plants, achieving around $60 \%$ of water soil content after 42 days of treatment. The exception was DHN11 plant \#9, which showed an intermediate rate between wild-type and transgenic plants, especially after 21 days of treatment. Despite these differences, similar water soil contents in all plant pots were obtained after 7 days of rehydration (inset, Fig. 5). All plants flowered during the experiment. None of the wild-type plants survived the stress assay, while all transgenic lines were able to cope with the imposed water deficit (Fig. 4b). After the stress treatment, all transgenic lines followed their normal growth cycle, producing fertile flowers, siliques, and seeds.

\section{Discussion}

Several studies in many plant species, including apple (Liang et al. 2012; Falavigna et al. 2015), have reported the relationship between dehydrins (DHNs) and several plant adaptive responses involving dehydration, especially seed development and abiotic stresses (reviewed in Hanin et al. 2011; Graether and Boddington 2014; Banerjee and Roychoudhury 2016). Specifically in apple, previous studies from our group suggested that MdoDHN11 is one of the proteins recruited to protect seeds during development (Falavigna et al. 2015). However, this hypothesis has not yet been evaluated. Within this context, we performed the functional characterization of MdoDHN11.

All main seed components, i.e., embryo, endosperm, nucellus and testa, were identified in apple seeds (Fig. 1). In apple, about 4-6 weeks after fertilization, the endosperm becomes cellular and soon fills much of the developing ovule



Fig. 3 Subcellular localization of MdoDHN11 in Arabidopsis leaf protoplasts. Negative and positive controls were composed by untransformed cells and cells transformed with $35 \mathrm{~S}:: E G F P$, respec- tively. All images were captured with a confocal laser scanning system. Green EYFP or EGFP, red chlorophyll, blue DAPI (color figure online) 



Fig. 4 Evaluation of transgenic Arabidopsis plants expressing MdoDHN11. a Real-time PCR of MdoDHN11 expression in Arabidopsis leaves. Gene expression was plotted relatively to DHN11 \#8. Standard error bars are shown. $n d$ not-detected. b Phenotype of trans- genic and wild-type plants exposed to water-deficit stress. The stress treatment was composed of removing the water supply for 6 straight weeks, followed by rewatering for 1 additional week (color figure online) 




$\leadsto$ WT \#1 $\rightarrow$ WT \#2 $\rightarrow$ DHN11 \#4 $\rightarrow$ DHN11 \#5 $\rightarrow$ DHN11 \#6 $\rightarrow$ DHN11 \#8



Fig. 5 Quantification of water loss rates of each pot. Pot capacity was maintained during the unstressed growing condition. The water deficit treatment consisted in removing the water supply of 2-month-old wild-type and transgenic plants for 42 days. For rehydration, water influx was allowed through diffusion. Inset shows the recovery phase (color figure online)

as it grows at the expense of the nucellus. Afterwards, the embryo gradually consumes the endosperm and occupies most of the seed (Dennis 2003). Embryo development from globular to torpedo, at the expense of the endosperm, was clearly visible through the stages analyzed (Fig. 1). To the best of our knowledge, the photomicrographs of the morphoanatomical analyses are, up to now, the best images reporting apple seed development in the literature, especially considering seeds from $40 \mathrm{~mm}$ fruits. These images may help the better understanding of analyses such as the ISH assays presented here.

MdoDHN11 transcripts accumulated in the nucellus layer, in the border with the endosperm of fruits with $40 \mathrm{~mm}$ diameter (Fig. 2). Due to the maternal origin of this layer, the accumulation of MdoDHN11 may represent a protective source for the embryo and the endosperm during seed development. Within this context, DHNs, as IDPs, are able to shift their conformational status under limited water availability and bind partly dehydrated surfaces of other proteins, protecting stored proteins and nutrients to support seed survival during maturation and desiccation (Tompa et al. 2006; Graether and Boddington 2014). Such activity would need the location of MdoDHN11 to be in the cytosol, which was demonstrated in our subcellular localization assays (Fig. 3). Recently, a subcellular localization study of all Arabidopsis DHN proteins showed that these proteins are localized in the cytosol or in the cytosol and nucleus (Candat et al. 2014). Phylogenetic analyses of DHNs from seven species, including apple and Arabidopsis, demonstrated that MdoDHN11 is present in a seed-specific clade together with Arabidopsis AtDHN14 and AtDHN45 (Falavigna et al. 2015). Interestingly, these two AtDHNs have the same subcellular localization of MdoDHN11, nucleus and cytosol (Candat et al. 2014). However, the nuclear localization is likely to be due to diffusion rather than targeting to nucleus (Candat et al. 2014). Taken together, these results fit well with our subcellular localization data and add additional evidence of the protective role of MdoDHN11 during apple seed development.

To gain insights about the MdoDHNII capacity to confer protection during restricted water availability, the MdoDHN11 gene was constitutively expressed in Arabidopsis and these plants were submitted to a severe water deficit assay. During the treatment, wild-type plants showed higher water loss rates than transgenic lines (Fig. 5). Similar findings were observed in water-deficit stress studies using transgenic plants ectopically expressing $D H N$ s from Medicago truncatula, Musa acuminata and Prunus mume (Shekhawat et al. 2011; Xie et al. 2012; Bao et al. 2017). The slow water losses showed by transgenic lines may be partly explained by their lower leaf area (Fig. 4b), which can consequently lead to a lower transpiration rate. However, no clear relationship could be drawn between water loss rate and total plant leaf area (Figs. 4, 5). As an example, DHN11 plant \#4 had the slowest water loss but was not the smallest plant. Another explanation may be directly linked to the DHN ability to bind water, which would help the maintenance of the original cell volume, preventing massive water losses and thus cellular collapse (Hanin et al. 2011).

The sole plants able to survive the water deficit treatment were the ones expressing the MdoDHN11 transgene (Fig. 4). Interestingly, all transgenic plants fully recovered less than $2 \mathrm{~h}$ after rehydration, suggesting that MdoDHN11 was able to maintain root integrity and functionality. Other reports have shown better root growth and development of plants constitutively expressing $D H N$ s in comparison to wild-type plants under water-deficit stress-inducing agents such as PEG or mannitol (Shekhawat et al. 2011; Yang et al. 2014). Studies with $D H N$ genes from several other plant species also pointed out the DHN ability to enhance tolerance to water deficit and other osmotic stresses (Cheng et al. 2002; Xing et al. 2011; Shekhawat et al. 2011; Xie et al. 2012; Ruibal et al. 2012; Yang et al. 2014; Bao et al. 2017). However, it is worth to mention that constitutive expression of $D H N$ s in plants is not a guarantee of increased tolerance to abiotic stresses (Hanin et al. 2011). As an example, transgenic tobacco plants ectopically expressing a Craterostigma plantagineum $D H N$ did not show increased drought tolerance (Iturriaga et al. 1992). One possible explanation is that the introduced gene was not sufficient to increase osmoprotection and may only work in cooperation with other molecules (Iturriaga et al. 1992). Indeed, overexpression of dehydrin $R A B 18$ in Arabidopsis did not improve stress tolerance, while its co-expression with dehydrin $\operatorname{Cor} 47$ increased freeze tolerance (Puhakainen et al. 2004).

The improved water deficit tolerance showed by transgenic Arabidopsis plants expressing MdoDHNII is evidence 
that MdoDHN11 is an important player in the plant response to limited water availability (Fig. 4). However, we cannot rule out that other MdoDHNs, especially MdoDHN1 (Supplementary Fig. 1), may also contribute for this trend during seed development. The protective role of DHNs may occur due to their disordered protein structure allied with their inability to denature (Graether and Boddington 2014). During dehydration, these features allow them to prevent massive water losses as well as to bind to other proteins and to interact with membranes, protecting tissues and cells under stress (Tompa et al. 2006; Kovacs et al. 2008; Banerjee and Roychoudhury 2016). If we consider the seed-specific expression of $M d o D H N 11$ confined to the nucellus layer, its probable function during apple seed development may be related to protect embryo and endosperm from limited water availability. Most likely, MdoDHN11 mechanism of action involves its interaction with proteins and other components present in the cell.

Acknowledgements Financiadora de Estudos e Projetos (0107009700); Embrapa (02.12.12.003.00.02); Conselho Nacional de Desenvolvimento Científico e Tecnológico (305549/2016-2 to GP, 308963/2015-6 to JEAM, 304991/2014-7 to MMP); Coordenação de Aperfeiçoamento de Pessoal de Nível Superior to VSF and JM; Fundação de Amparo à Pesquisa do Estado do Rio Grande do Sul to CPS. We thank Dr. Henrique P. dos Santos (Embrapa Uva e Vinho) for providing us with the access to apple orchards; Dr. Marcelo C. Dornelas (UNICAMP) for his help in the ISH assay; and Juliana Troleis (UFRGS) for her help in using the microscope photography apparatus. We also thank our colleagues at Laboratory of Plant Molecular Genetics (Embrapa Uva e Vinho), especially Dr. Rafael Augusto Arenhart, for technical assistance.

Author contribution statement VSF, LFR, MMP and GP conceived the work and the experimental design; VSF, JM, VB and JEAM performed the ISH and the seed morphological assays; VSF and CPS performed the subcellular localization assay; VSF performed field samplings, nucleic acid extractions, real-time PCR assays, gene amplifications and cloning, Arabidopsis transformation, water deficit assay, and drafted the manuscript; LFR, MMP and GP revised the manuscript.

\section{Compliance with ethical standards}

Conflict of interest The authors declare that they have no conflict of interest.

\section{References}

Banerjee A, Roychoudhury A (2016) Group II late embryogenesis abundant (LEA) proteins: structural and functional aspects in plant abiotic stress. Plant Growth Regul 79:1-17. https://doi. org/10.1007/s10725-015-0113-3

Bao F, Du D, An Y et al (2017) Overexpression of Prunus mume dehydrin genes in tobacco enhances tolerance to cold and drought. Front Plant Sci 8:151. https://doi.org/10.3389/fpls.2017.00151

Bleckmann A, Alter S, Dresselhaus T (2014) The beginning of a seed: regulatory mechanisms of double fertilization. Front Plant Sci 5:452. https://doi.org/10.3389/fpls.2014.00452
Candat A, Paszkiewicz G, Neveu M et al (2014) The ubiquitous distribution of late embryogenesis abundant proteins across cell compartments in Arabidopsis offers tailored protection against abiotic stress. Plant Cell 26:3148-3166. https://doi.org/10.1105/ tpc. 114.127316

Cheng Z, Targolli J, Huang X, Wu R (2002) Wheat LEA genes, PMA80 and PMA1959, enhance dehydration tolerance of transgenic rice (Oryza sativa L.). Mol Breed 10:71-82

Clough SJ, Bent AF (1998) Floral dip: a simplified method for Agrobacterium-mediated transformation of Arabidopsis thaliana. Plant J 16:735-743

Dennis F (2003) Flowering, pollination and fruit set and development. In: Ferree D, Warrington I (eds) Apples botany, production and uses. CAB International, Wallingford, pp 153-165

Falavigna VS, Porto DD, Buffon V et al (2014) Differential transcriptional profiles of dormancy-related genes in apple buds. Plant Mol Biol Report 32:796-813. https://doi.org/10.1007/ s11105-013-0690-0

Falavigna VS, Miotto YE, Porto DD et al (2015) Functional diversification of the dehydrin gene family in apple and its contribution to cold acclimation during dormancy. Physiol Plant 155:315329. https://doi.org/10.1111/ppl.12338

Falavigna VS, Porto DD, Miotto YE et al (2018) Evolutionary diversification of galactinol synthases in Rosaceae: adaptive roles of galactinol and raffinose during apple bud dormancy. J Exp Bot 69:1247-1259. https://doi.org/10.1093/jxb/erx451

Feder N, O’brien T (1968) Plant microtechnique, some principles and new methods. Am J Bot 55:123-142

Gerrits P, Smid L (1983) A new less toxic polymerization system for the embedding of soft tissue in glycol methacrylate and subsequent preparing of serial sections. J Microsc 132:81-85

González-Morales SI, Chávez-Montes RA, Hayano-Kanashiro C et al (2016) Regulatory network analysis reveals novel regulators of seed desiccation tolerance in Arabidopsis thaliana. Proc Natl Acad Sci 113:E5232-E5241. https://doi.org/10.1073/ pnas. 1610985113

Graether SP, Boddington KF (2014) Disorder and function: a review of the dehydrin protein family. Front Plant Sci 5:576. https:// doi.org/10.3389/fpls.2014.00576

Hanin M, Brini F, Ebel C et al (2011) Plant dehydrins and stress tolerance: versatile proteins for complex mechanisms. Plant Signal Behav 6:1503-1509. https://doi.org/10.4161/psb.6.10.17088

Hartmann H, Kester D, Davies F, Geneve R (2010) Hartmann and Kester's plant propagation: principles and practices, 8th edn. Prentice Hall, Upper Saddle River

Iturriaga G, Schneider K, Salamini F, Bartels D (1992) Expression of desiccation-related proteins from the resurrection plant $\mathrm{Cra}$ terostigma plantagineum in transgenic tobacco. Plant Mol Biol 20:555-558. https://doi.org/10.1007/BF00040614

Karimi M, Depicker A, Hilson P (2007) Recombinational cloning with plant Gateway vectors. Plant Physiol 145:1144-1154. https ://doi.org/10.1104/pp.107.106989

Kovacs D, Kalmar E, Torok Z, Tompa P (2008) Chaperone activity of ERD10 and ERD14, two disordered stress-related plant proteins. Plant Physiol 147:381-390. https://doi.org/10.1104/ pp. 108.118208

Lefort F, Douglas GC (1999) An efficient micro-method of DNA isolation from mature leaves of four hardwood tree species Acer, Fraxinus, Prunus and Quercus. Ann For Sci 56:259-263. https ://doi.org/10.1051/forest:19990308

Liang D, Xia H, Wu S, Ma F (2012) Genome-wide identification and expression profiling of dehydrin gene family in Malus domestica. Mol Biol Rep 39:10759-10768. https://doi.org/10.1007/ s11033-012-1968-2 
Liu Y, Song Q, Li D et al (2017) Multifunctional roles of plant dehydrins in response to environmental stresses. Front Plant Sci 8:1018. https://doi.org/10.3389/fpls.2017.01018

Lodhi MA, Ye G-N, Weeden NF, Reisch BI (1994) A simple and efficient method for DNA extraction from grapevine cultivars and Vitis species. Plant Mol Biol Report 12:6-13. https://doi. org/10.1007/BF02668658

Malabarba J, Buffon V, Mariath JEA et al (2017) The MADS-box gene Agamous-like 11 is essential for seed morphogenesis in grapevine. J Exp Bot 68:1493-1506. https://doi.org/10.1093/jxb/erx025

McDowell E, Trump B (1976) Histological fixatives for diagnostic light and electron microscopy. Arch Pathol Lab Med 1000:405-414

Puhakainen T, Hess MW, Mäkelä P et al (2004) Overexpression of multiple dehydrin genes enhances tolerance to freezing stress in Arabidopsis. Plant Mol Biol 54:743-753. https://doi.org/10.1023/ B:PLAN.0000040903.66496.a4

Riley AC, Ashlock DA, Graether SP (2019) Evolution of the modular, disordered stress proteins known as dehydrins. PLoS One 14:e0211813. https://doi.org/10.1371/journal.pone.0211813

Ruibal C, Salamó IP, Carballo V et al (2012) Differential contribution of individual dehydrin genes from Physcomitrella patens to salt and osmotic stress tolerance. Plant Sci 190:89-102. https://doi. org/10.1016/j.plantsci.2012.03.009

Shekhawat UKS, Srinivas L, Ganapathi TR (2011) MusaDHN-1, a novel multiple stress-inducible SK3-type dehydrin gene, contributes affirmatively to drought- and salt-stress tolerance in banana. Planta 234:915-932. https://doi.org/10.1007/s00425-011-1455-3

Sreenivasulu N, Wobus U (2013) Seed-development programs: a systems biology-based comparison between dicots and monocots. Annu Rev Plant Biol 64:189-217. https://doi.org/10.1146/annur ev-arplant-050312-120215

Tompa P, Bánki P, Bokor M et al (2006) Protein-water and proteinbuffer interactions in the aqueous solution of an intrinsically unstructured plant dehydrin: NMR intensity and DSC aspects. Biophys J 91:2243-2249. https://doi.org/10.1529/bioph ysj.106.084723

Wu F-H, Shen S-C, Lee L-Y et al (2009) Tape-Arabidopsis sandwicha simpler Arabidopsis protoplast isolation method. Plant Methods 5:16. https://doi.org/10.1186/1746-4811-5-16

Xie C, Zhang R, Qu Y et al (2012) Overexpression of MtCAS31 enhances drought tolerance in transgenic Arabidopsis by reducing stomatal density. New Phytol 195:124-135. https://doi.org/1 0.1111/j.1469-8137.2012.04136.x

Xing X, Liu Y, Kong X et al (2011) Overexpression of a maize dehydrin gene, $Z m D H N 2 b$, in tobacco enhances tolerance to low temperature. Plant Growth Regul 65:109-118. https://doi. org/10.1007/s10725-011-9580-3

$\mathrm{Xu}$ W, Fiume E, Coen O et al (2016) Endosperm and nucellus develop antagonistically in Arabidopsis seeds. Plant Cell 28:1343-1360. https://doi.org/10.1105/tpc.16.00041

Yang Y, Sun X, Yang S et al (2014) Molecular cloning and characterization of a novel SK3-type dehydrin gene from Stipa purpurea. Biochem Biophys Res Commun 448:145-150. https://doi. org/10.1016/j.bbrc.2014.04.075

Zeng Y, Yang T (2002) RNA isolation from highly viscous samples rich in polyphenols and polysaccharides. Plant Mol Biol Report 20:417. https://doi.org/10.1007/BF02772130

Publisher's Note Springer Nature remains neutral with regard to jurisdictional claims in published maps and institutional affiliations. 